EPJ Web of Conferences 19, 10007 (2012)

DOI: $10.1051 /$ epjconf/20121910007

(C) Owned by the authors, published by EDP Sciences, 2012

\title{
A detailed self-consistent vertical Milky Way disc model
}

\author{
A. Just ${ }^{\mathrm{a}}$, O. Golubov and S. Gao \\ Astronomisches Rechen-Institut, Zentrum für Astronomie der Universität Heidelberg, \\ Mönchhofstraße 12-14, 69120 Heidelberg, Germany
}

\begin{abstract}
We present a self-consistent vertical disc model of thin and thick disc in the solar vicinity. The model is optimized to fit the local kinematics of main sequence stars by varying the star formation history and the dynamical heating function. The star formation history and the dynamical heating function are not uniquely determined by the local kinematics alone. For four different pairs of input functions we calculate star count predictions at high galactic latitude as a function of colour. The comparison with North Galactic Pole data of SDSS/SEGUE leads to significant constraints of the local star formation history.
\end{abstract}

\section{INTRODUCTION}

In [2] we published a self-consistent vertical disc model of thin and thick disc in the solar cylinder. The thin disc model is based on a series of stellar subpopulations with surface densities and velocity dispersions according to an adopted star formation history (SFR) and a dynamical heating function (AVR). An isothermal thick disc component is included self-consistently. The discs are in dynamical equilibrium including the gravitational forces of the gas component and the dark matter halo. A simple chemical enrichment model is included to reproduce the metallicity distribution of the G-dwarfs in the solar neighbourhood. The SFR and AVR are varied in order to fit the local kinematics of main sequence (MS) stars and to match other constraints of the local stellar disc. We selected four models fitting the local kinematics equally well, but the SFR cannot be determined by the local kinematics alone (top panels of Fig. 1).

\section{LOCAL NORMALIZATION}

For a comparison of the model predictions with star counts of the Sloan Digital Sky Survey (SDSS) we need the properties of the MS in the solar neighbourhood in the $u, g, r, i, z$ filter system. We applied filter transformations from $B, V, R, I$ to $u, g, r, i, z$ to the local sample ([1]). The absolute magnitudes $M_{g}(g-r)$ for the thin disc, thick disc and stellar halo are used to calculate the apparent magnitude distribution vertical to the Galactic plane. Since the number densities $N_{25}(g-r)$ in a $25 \mathrm{pc}$ sphere are very noisy and the relative contributions of the components are not well known, we use these numbers for each component as free fitting parameters (middle panels of Fig. 1).

\section{NGP DATA AND THE SFR}

The local model predicts vertical density profiles as a function of lifetime (or colour) of the MS stars but the profiles of models A-D differ significantly from a sech ${ }^{2}$ model and from each other. We use

ae-mail: just@ari.uni-heidelberg.de

This is an Open Access article distributed under the terms of the Creative Commons Attribution-Noncommercial License 3.0, which permits unrestricted use, distribution, and reproduction in any noncommercial medium, provided the original work is properly cited. 

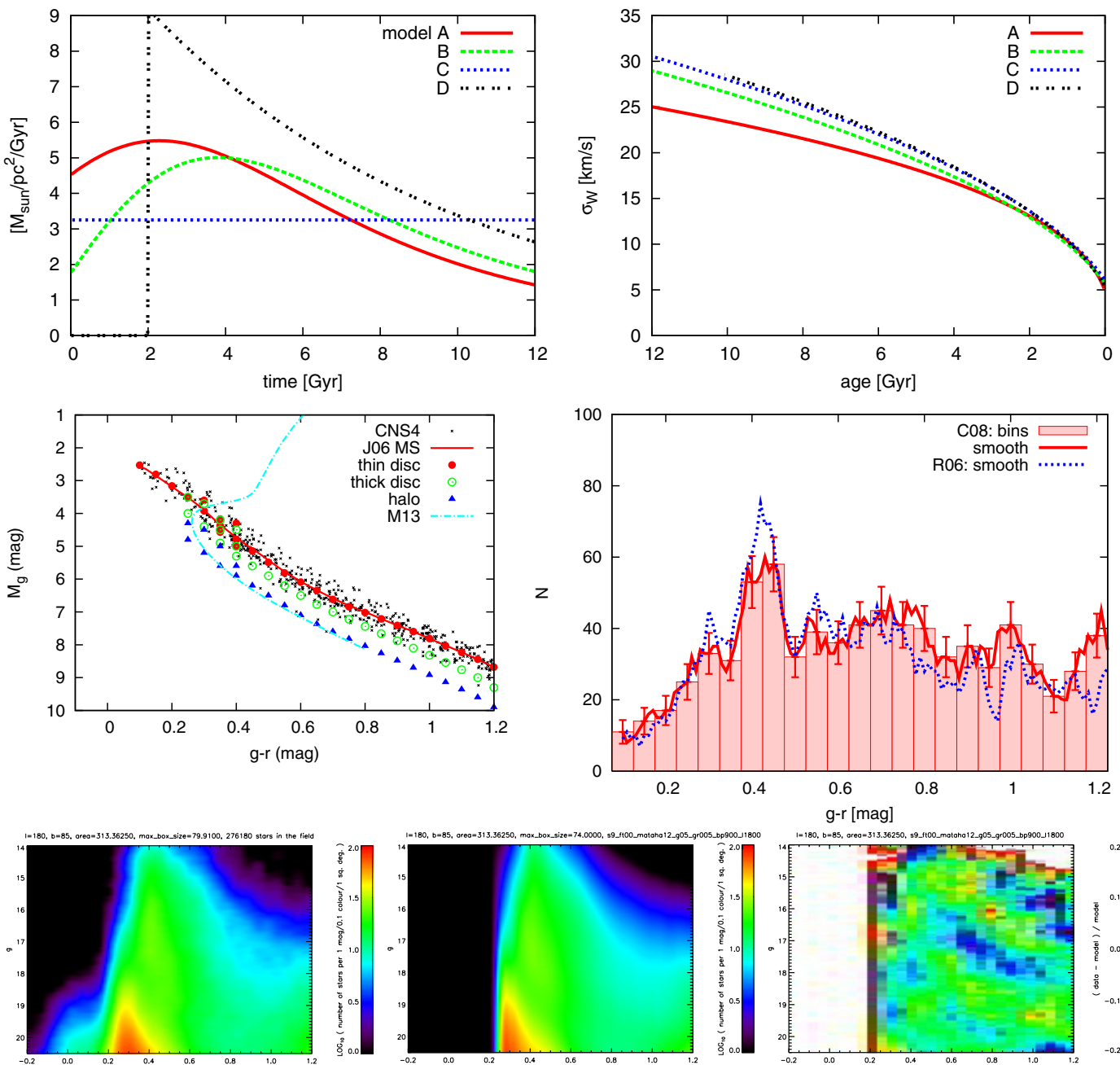

Figure 1. Top panels: SFRs (left) and AVRs (right) of models A-D. Middle panels: Local MS of thin disc, thick disc, and stellar halo with MS stars of the CNS4 and the fiducial sequence of M13 $([\mathrm{Fe} / \mathrm{H}]=-1.53)$ overplotted (left). Local number density in the $25 \mathrm{pc}$ sphere in $g-r$ bins (right). Bottom panels: Smoothed Hess diagrams (number counts per $\mathrm{deg}^{2}$, per mag in $\mathrm{g}$ and $0.1 \mathrm{mag}$ in g-r in log-scale between 1 and 100) of SDSS data (left) and model A (middle) at the NPG field; Relative differences (data - model)/model in the range of $\pm 20 \%$ (right).

star counts of SDSS (DR7) for the North Polar Cap (NGP) field with $b>80^{\circ}$ and construct the Hess diagram in (g-r,g) applying standard dereddening ([3]). Similar Hess diagrams of models A-D were calculated with a best fit algorithm of the local normalization in each colour bin including a flattened power law stellar halo. A $\chi^{2}$ analysis shows that model A matches the NGP data significantly better than the other models. Model A fits the data better than \pm 10 percent and is the best model to reproduce the local kinematics and the observed star counts of SDSS in the NGP field (bottom panels of Fig. 1).

\section{References}

[1] Just, A., Jahreiß, H., AN, 329, (2008) 790-800

[2] Just, A., Jahreiß, H., MNRAS, 402, (2010) 461-478

[3] Just, A., Gao, S., Vidrih, S., MNRAS, 411, (2011) 2586-2595 